\title{
Mehrsprachigkeit in Wiens Straßen und Märkten
}

\section{Multilingualism in Vienna's streets and markets}

\section{Thomas Fritz}

\begin{abstract}
:
This paper starts with a short debate on the role of the research method of "linguistic landscapes", which is part of the ethnographic paradigm. It presents multilingualism, or rather metrolingualism in the market place; a space where languages meet and intermingle and multilingual repertoires collaborate to make linguistic actions possible. The socio-demographic element of linguistic landscapes is also shown in the existence of China Town in Vienna. Multilingualism "from below" is contrasted to the official language policies of the country.
\end{abstract}

\section{Keywords:}

Multilingualism; metrolingualism; Linguistic Landscapes; multilingual repertoires 
In diesem Beitrag wird der Forschungsansatz der Linguistic Landscapes, der einen Teil des ethnographischen Forschungsparadigmas darstellt, kurz in seiner Bedeutung für die Soziolinguistik umrissen und anhand von Daten aus einem Linguistic Landscape Projekt die Mehrsprachigkeit in Wien in einigen Bezirken und Bereichen (z.B. Märkten) dargestellt. Mehrsprachigkeit bedeutet in diesem Kontext die unregulierte, informelle Verwendung von plurilingualen Repertoires im täglichen Handeln.

Bei Linguistic Landscape handelt es sich um einen ethnographischen Ansatz, dessen Ziel es ist, die Mehrsprachigkeit eines geografischen Bereichs, also eines Stadtviertels, einer Straße, eines Gebäudes, zum Beispiel eines Bahnhofs, zu zeigen mit dem Ziel, die durch die sichtbaren Zeichen dokumentierte soziale Beschaffenheit dieses Ortes sichtbar zu machen.

"[m]essages in the public space are never neutral, they always display connections to social structure, power and hierarchies [...]." (Blommaert 2013: 40)

Verschiedene gesellschaftliche Gruppen zeigen durch Beschriftungen im öffentlichen Raum ihre Präsenz; zentral ist hier die Fragestellung, „wer darf wo etwas schreiben“. Durch einen genaueren, beobachtenden Blick auf die Mehrsprachigkeit von „unten“, also einer unreglementierten Präsenz von Sprachen, erkennen wir die regional verschiedene Anwesenheit von Gruppen, die oftmals im öffentlichen Raum sonst keine Stimme erhalten. Linguistic Landscape hat immer auch eine historische, chronotopische Dimension (vgl. Blommaert 2018), die Präsenz der Sprachen an bestimmten Orten ändert sich und spiegelt aktuelle und vergangene Ereignisse wieder.

Dort, wo der formale Charakter der Sprachverwendung, wie zum Beispiel bei öffentlichen Beschilderungen, nicht mehr zum Tragen kommt, können wir lebendiges, buntes und sprachlich vielfältiges Sprachenleben beobachten.

Der ethnographische Forschungsansatz der Linguistic Landscape hat in den letzten Jahren einerseits viel an Attraktivität gewonnen, ist im so genannten Mainstream angekommen und hat jedoch dadurch viel von seinem ursprünglichen Charakter als soziolinguistisch ausgerichteter Forschungszweig verloren. In dem in diesem Beitrag vorgestellten Projekt wurde einerseits der von Blommaert eingemahnten soziolinguistischen Ausrichtung von Linguistic Landscape Rechnung getragen, also dem „ethnographic understanding of social structure as dynamic, fragmented and essentially stochastic, i.e. chaotic“ (Blommaert 2016: 4) und andererseits wurde unter Einbeziehung des Konzepts des Metrolingualismus (vgl. Pennycook Otsuji 2015) die Vermischung von sprachlichen Ressourcen in konkreten Sprachhandlungen wahrgenommen, hier manifestiert durch sichtbare Zeichen. Metrolingualismus bedeutet kurz zusammengefasst das Verwenden aller zur Verfügung stehender Ressourcen um konkrete Sprachhandlungen vor allem im urbanen Raum, der durch das Nebeneinander verschiedener sprachlicher Repertoires (vgl. Busch 2013: 20 und Blommaert und Backus 2013) gekennzeichnet ist. Die Dynamik von Repertoires und die daraus resultierenden „überraschenden“ Effekte sind vor allem im unreglementierten, die formalen Sprachenregime ignorierenden Raum der Märkte sichtbar. 


\section{Ein Überblick - die quantitative Perspektive}

Seit 2015 werden vom Verfasser dieses Beitrags und von Projektmitarbeiter*innen, Studierenden an der Universität Wien und anderen Interessierten, Fotos zur sprachlichen Vielfalt aufgenommen und dokumentiert. Mittlerweile besteht der gesamte Datensatz aus ca. 7.000 Bildern aus verschiedenen Bereichen Wiens: dem 16. Bezirk, hier vor allem dem Brunnenmarkt, dem 10. Bezirk, vor allem dem Viktor Adler Markt, dem Hannover Markt im 20. Bezirk und aus dem vierten Bezirk, der sich in der Nähe des Stadtzentrums befindet.

Ein rein quantitativer Blick ergibt, dass in diesen Bereichen schriftliche Spuren von mehr als 30 Sprachen gefunden wurden. Nicht verwunderlich ist die Mehrsprachigkeit besonders auf den Märkten stark vertreten, da Märkte Orte einer dynamischen Koexistenz verschiedener Menschen und damit verschiedener Sprachen sind. Märkte sind Orte, an denen ,[...] the changing, shifting world of the urban landscape, where languages are blended, sorted, created, used for new purposes, taken up, tossed aside, learned and renewed." (Pennycook und Otsuji 2015: 30). Gleichzeitig sind Märkte öffentliche Orte, an denen Präsenz, Sichtbarkeit, soziale Hierarchien und Macht verhandelt werden:

"The public space of a market square or a highway is, in contrast to the private space of, for example one's dining room, a shared space over which multiple people and groups will try to acquire authority and control, if not over the whole of the space, then at least over parts of it." (Blommaert 2013: 40)

Nicht überraschend ergibt der quantitative Blick eine klare Dominanz des Deutschen, gefolgt von Beispielen, die ich in diesem Kontext metrolingual bezeichnen will, die also

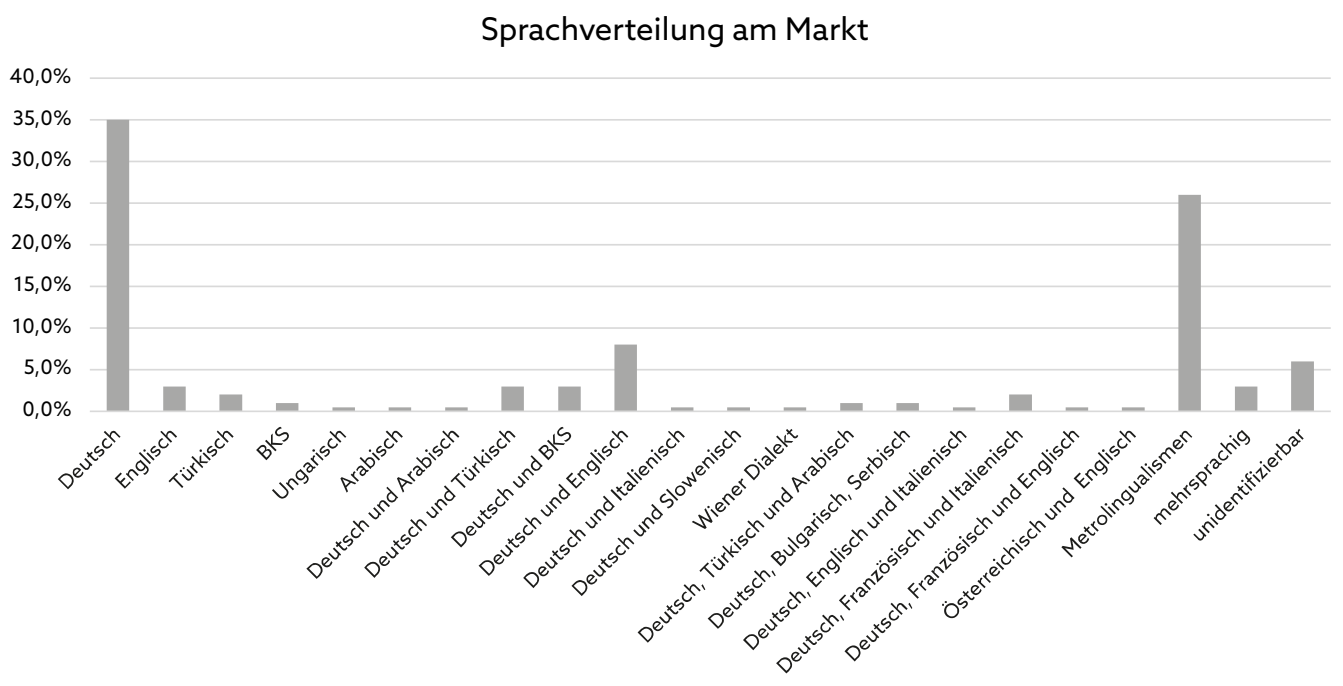

Abb. 1: Sprachenverteilung am Brunnenmarkt 
eine Mischung aus verschiedenen multilingualen Repertoires repräsentieren. Diese sind mit einem Anteil von ca. $26 \%$ sehr hoch und nicht nur vereinzelt auftretende Phänomene. Wir erkennen folglich, dass, das, was Pennycook und Otsuji (2015) das Jonglieren mit Sprachen nennen, auf dem Markt eine Art Normalität darstellt.

\section{Der Blick aufs Detail - die qualitative Perspektive}

Aus der obigen Übersicht erkennen wir, dass Märkte die Orte sind, an denen sich sprachliche Vielfalt zeigt, sehr oft in Kombination mit Deutsch, aber auch mit anderen Sprachen. Insgesamt können wir 37 Sprachen identifizieren, wobei die klare Abgrenzung zwischen einzelnen Sprachen nicht immer möglich ist, so wie das Konzept Sprache, wenn es sich nicht einer - oftmals national geprägten Sichtweise - verschließt, auch in Frage gestellt werden muss (vgl. Busch 2013: 107f). Die Sprachen, die bei der Erhebung festgestellt wurden sind:

Afghanisch, Albanisch, Arabisch, BKS, Bulgarisch, Chinesisch, Deutsch, Englisch, Finnisch, Französisch, Griechisch, Hebräisch, Hindi, Italienisch, Japanisch, Koreanisch, Kroatisch, Kurdisch, Latein, Mandarin, Niederländisch, Österreichisch, Paschtu/Dari, Polnisch, Portugiesisch, Russisch, Serbisch, Slowakisch, Spanisch, Tahitianisch, Thai, Türkisch, Tschechisch, Tschetschenisch, Ungarisch, Vietnamesisch, Wienerisch.

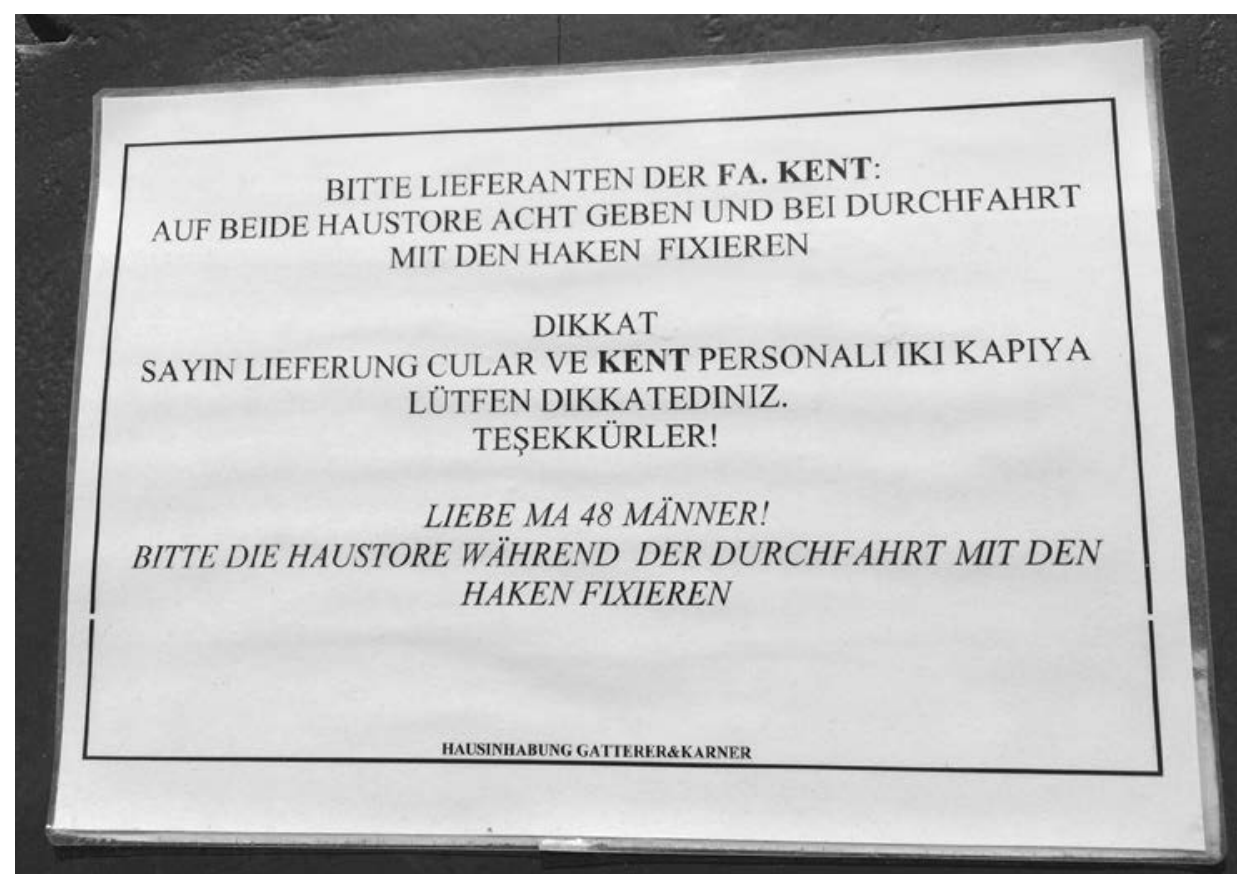

Abb. 2: Schild Kent 
In dieser Aufzählung fällt auf, dass sowohl „Österreichisch“ als auch „Wienerisch“ angeführt werden, was einerseits einem nicht-nationalsprachlichen Verständnis von Sprache entspricht und andererseits zeigt, dass der Umgang mit Sprachen oftmals ein spielerischer, nicht ganz ernst gemeinter und auf jeden Fall ein normverstoßender ist.

Im Folgenden sollen einige Beispiele gezeigt werden, die die sprachliche Vielfalt und Kreativität repräsentieren.

Ein Schild beim Lieferanteneingang eines türkischen Lokals (Kent) adressiert einerseits deutschsprechende Lieferant*innen und andererseits türkischsprachige. Eine Gruppe, die der MA 48 Männer, d.h. die Müllabfuhr, die in Wien informell die 48-er genannt werden, wird eigens angesprochen. Bemerkenswert ist auch, dass im türkischen Text ein „Danke“ formuliert wird, das in dem deutschen Text fehlt.

Die gleichzeitige Präsenz mehrerer Sprachen ist auch in diesem Bild zu sehen, Eingang und Giris, Deutsch und Türkisch.



Abb. 3: Schild Brunnenmarkt

Die Vielfalt der Märkte lässt sich nicht nur an ihrer Vielsprachigkeit erkennen, sondern auch durch eine Multifunktionalität der Stände, die nicht nur ein Produkt anbieten und anpreisen, sondern eine breite Palette an Produkten. Dies ist auf mehrere Umstände zurückzuführen; einerseits auf den Wunsch denn Kund*innen mehr als eine Sache anzubieten und daher das Service zu erhöhen und andererseits auf die ökonomische Notwendigkeit der Diversifizierung, die es ermöglichen soll, im Konkurrenzkampf mit den anderen Anbietern bestehen zu können.

Diese Multifunktionalität der Marktstände ist auf dem folgenden Bild zu erkennen, das in einer Fleischerei aufgenommen wurde, ganz explizit aber Werbung für einen 
Internetprovider macht, und in mehreren Sprachen angibt, dass die Produkte "halal“ sind, eine wichtige Information für Kund*innen mit muslimischen Essensvorschriften. Wir erkennen hier auch die Adressierung spezieller Kund*innengruppen, also eine Spezialisierung für unterschiedliche „kulturelle“ Anforderungen, denn nicht nur die Marktstände sind divers, auch die Menschen, die auf Märkten einkaufen.

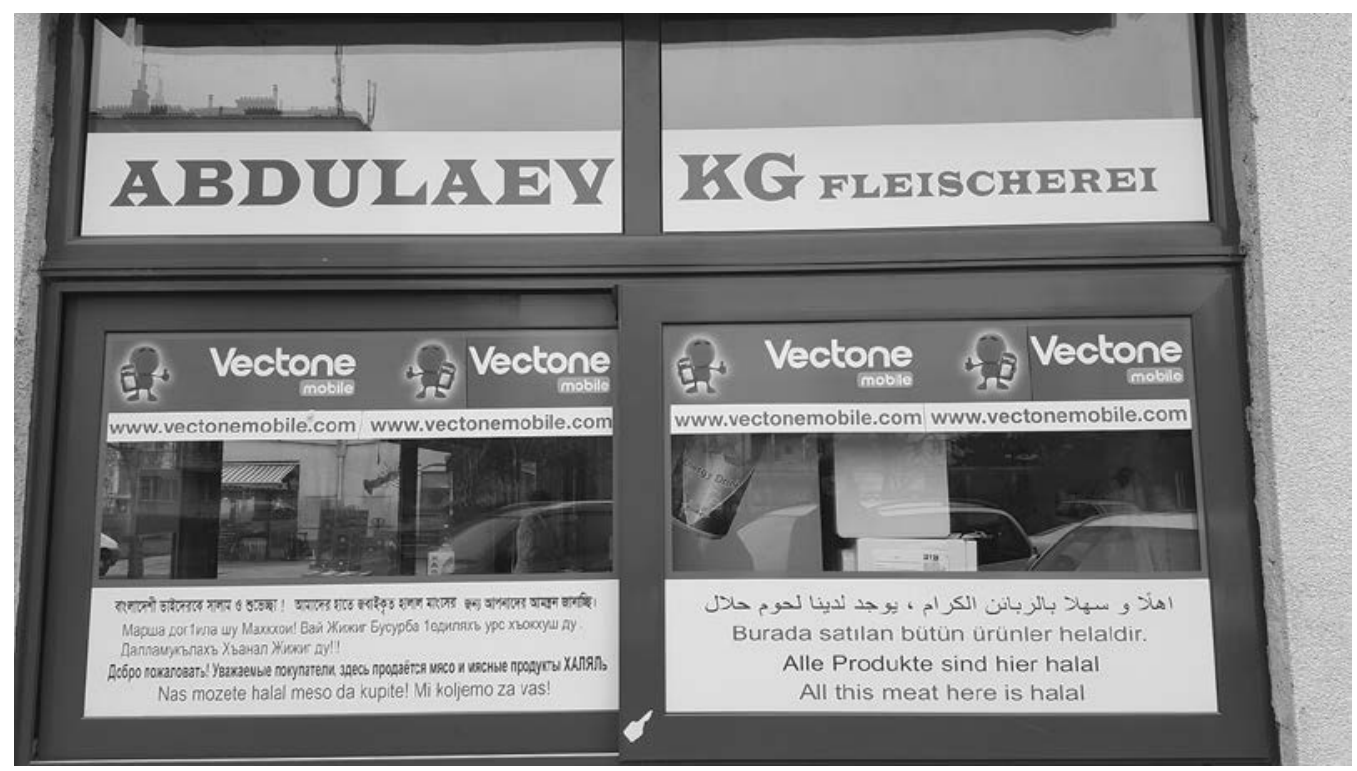

Abb. 4: Marktstand Brunnenmarkt

Diese Tatsache wird auch sichtbar, wenn auf einem Fischstand die Produkte in Türkisch und Deutsch angeboten werden, sich aber an diesem Stand im Jänner 2017 auch eine arabische Aufschrift befindet. Laut Auskunft des Betreibers, eines Syrers dient diese Aufschrift auf auch? dazu „meinen Leuten“ zu sagen, dass es hier frische Fische gibt, die geputzt und für die Zubereitung vorbereitet sind. (mündliche Kommunikation, Jänner 2017). Ähnliches gilt für einen Stand, an dem Hühner verkauft werden und eine Notiz auf Dari zu finden ist, wiederum eine eindeutige Adressierung einer besonderen Gruppe (mündliche Kommunikation, Jänner 2017).

Die Menschen, die auf den Märkten arbeiten, kommen aus den verschiedensten Kontexten, sprechen unterschiedliche Sprachen und werden daher auch unterschiedlich angesprochen, wie das Bild einer "Jobannonce“ zeigt, in der, wahrscheinlich von einem Kroatisch oder Bosnisch sprechenden Besitzer, ein Grillmeister gesucht wird.

Märkte sind also die Orte, an denen viele Sprachen nebeneinander existieren und damit unterschiedliche Menschen möglichst in „ihren“ Sprachen angesprochen werden sollen. Die Vielfalt der Sprachen spiegelt die Vielfalt der Menschen und die Diversität der Stadt wieder, dies auch in der gezeigten Intensität, weil diese Orte nicht von der offiziellen Sprachenpolitik reguliert werden. Wir sehen hier also das „wahre“ Gesicht einer Stadt in der laut Integrationsstadtrat 250 Sprachen gesprochen werden und in der 


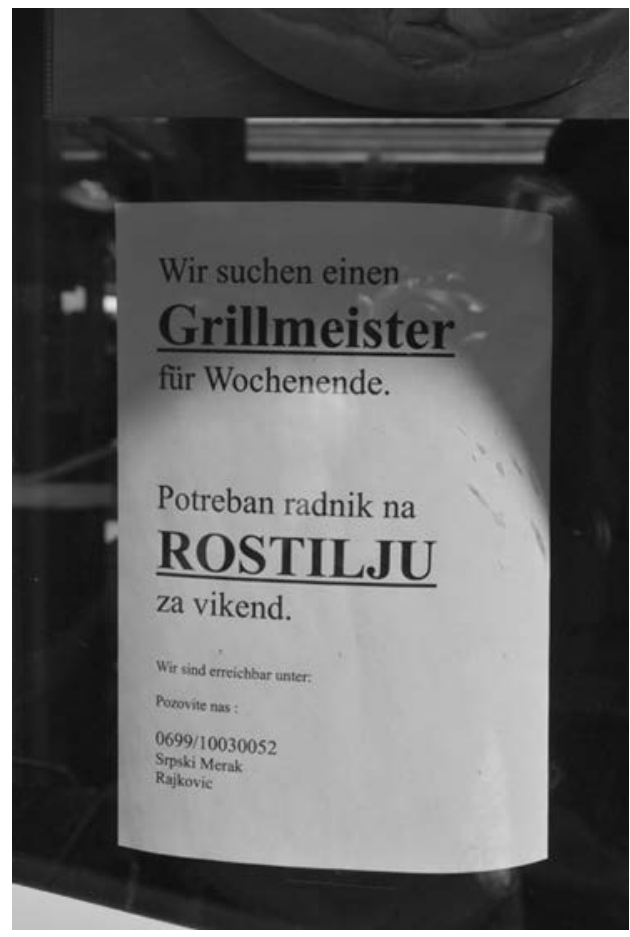

Abb. 5: Marktstand Hannovermarkt

mehr als $50 \%$ der Einwohner*innen einen so genannten Migrationshintergrund aufweisen (Stadt Wien 2019), einer Stadt also, die wir als superdivers (Vertovec 2007) bezeichnen können. Wir sehen hier deutlich einen „grassroots multilingualism“ - eine Mehrsprachigkeit also, die nicht offiziell anerkannt, dennoch aber sehr produktiv ist (vgl. Pennycook Otsuji 2015: 13).

Aus einer linguistischen Perspektive sind jedoch auch Beispiele interessant, in denen sich die sprachliche Vielfalt nicht in einem Nebeneinander von Sprachen manifestiert, sondern in einer Gleichzeitigkeit und einem Miteinander.

Eines der beeindruckendsten Beispiele ist der Sinitlah, der auf einem Gemüsestand am Brunnenmarkt gefunden wurde. Nehmen wir system-linguistische Kriterien zur Hand, so erkennen wir, dass die Schreibung des Wortes Schnittlauch hier zu einem großen Teil der türkischen Phonologie folgt. Im Türkischen sind Silben mit einer CCV Struktur nicht möglich, wie in vielen anderen Sprachen (vgl. Hayes 2009; Hall 2000). Mittels des Prozesses der Einfügung eines Sprossvokals, eines Prozesses, der auch Epenthese genannt wird, muss die Silbe der erstsprachigen Silbenstruktur angepasst werden. Daraus folgt sinitlah. Die Kombination /tl/ stellt kein Problem dar, weil es sich hier um Silbengrenzen handelt. Das Deutsche /ch/ wird im Türkischen durch ein einfaches /h/ repräsentiert. Damit ist das Wort fertig konstruiert, weist aber auch in der türkischen Rechtschreibung noch eine Normabweichung auf, das /s/ sollte ein /s/ sein.

Wir erkennen hier also, dass von der/dem Schreiber*in das in ihrem vorhandenen sprachlichen Repertoire vorhandene Wissen um „Deutsch“ und „Türkisch“ benützt wurde, um ein Wort zu produzieren, das nicht mehr eindeutig einer Sprache zugeordnet werden kann. Eine Auswahl aus den weiteren reichhaltigen Beispielen sind:

\author{
„mealige Kartoffel“ \\ „Petesiele“ \\ "Chuchini“ \\ "Gurgen“ \\ „Spinak“ \\ „fistkochende Kartofel“
}


Für alle Beispiele sind zwei Elemente bezeichnend, einerseits die oben bereits im Detail beschriebene „Kollaboration“ von Elementen verschiedener „Sprachen“ in einem metrolingualen Repertoire und andererseits die starke Orientierung an der mündlichen Sprache, also einem Register, das nicht von der Norm der Schriftlichkeit beeinflusst ist und damit der Vermischung von verschiedenen Registern in diesen Repertoires.

\section{Wer lebt wo in der Stadt? Soziographische Hinweise}

Linguistic Landscapes geben uns auch Hinweise darauf, welche Sprecher*innen welcher Sprachen in der Stadt leben und wie die soziographische Beschaffenheit eines Bezirkes ist. Die Daten aus dem 16. Bezirk, einem der traditionellen Arbeiter - und mittlerweile Migrant*innenbezirke zeigen, dass es neben dem vorherrschenden Deutsch auch andere Sprachen gibt, die relativ stark präsent sind. In Ottakring sind dies Türkisch und vor allem Serbisch, Kroatisch und Bosnisch. Vor allem in der Nähe des Brunnenmarkts und der Ottakringerstraße sehen wir eine große Präsenz der Sprachen des ehemaligen Jugoslawiens. Wir erkennen anhand von Graffitis, die Konflikte, die zwischen den einzelnen Gruppen ausgetragen werden, sehr oft über die Namen der jeweiligen Fußballklubs und ihrer teils sehr nationalistischen und rechten Fanclubs. Das Ustascha Zeichen ist ebenso zu finden wie die vier „S“, die die nationalistischen Serben benutzen. In anderen Bezirken, vor allem jenen, die keine Tradition in Bezug auf Arbeiterwohnsiedlungen und Migrant*innen besitzen, finden wir andere Sprachen, so zum Beispiel in Wieden, einem relativ zentralen Bezirk Wiens, der an die Innenstadt angrenzt und eine bürgerliche Tradition aufweist - und heute tendenziell von jungen aufsteigenden Menschen und Student*innen bewohnt wird. Hier sind fast keine türkischen Aufschriften zu sehen, in unserem Datensatz befindet sich lediglich eine Beschriftung aus Wieden. Jedoch sind hier viele asiatische Sprachen zu finden: Koreanisch, Japanisch und vor allem Chinesisch. Letzteres ist vor allem in Bezug auf die Frage, ob es in Wien eine Chinatown gibt, interessant. In einem Bereich von Wieden finden wir Restaurants, Haushaltswarengeschäfte, Reisebüros - seit einiger Zeit auch zwei Frisöre - einen Kulturverein, die Redaktion einer Zeitung und ein Bildungsinstitut, die Chinesisch beschriftet sind, zwei davon nur auf Chinesisch. Dies deutet eindeutig auf eine Adressierung der chinesischen Bevölkerung Wiens - und auf die Existenz einer China Town, wiewohl dies in Wien fast nicht bekannt ist. 2008 gab es eine Diskussion zwischen der Stadtverwaltung und der chinesischen Community, um die Errichtung eines der berühmten schönen roten chinesischen Tore. Dies wurde jedoch nie realisiert, weil die Community nicht so sichtbar sein wollte.

\section{Abschließende Gedanken}

Linguistic Landscapes sind als ethnographische Methode ein wichtiges und produktives Instrument, um Mehrsprachigkeit in ihrer schriftlich repräsentierten Form, und damit der Präsenz der Verortung und der Kämpfe um Raum einer urbanen Gesellschaft auf 
die Spur zu kommen. Die in diesem Beitrag beschriebene Mehrsprachigkeit „von unten“ könnte als widerständig gegen die einsprachige Norm, die von Staats wegen verordnet wird, gesehen werden - was sie mitunter auch ist. Sie ist sie aber auf jeden Fall, Normalität einer Gesellschaft, die lebendig, gemischt, kreativ und grenzüberschreitend ist, einer Gesellschaft also in der nicht mehr das Erwartbare sichtbar ist, sondern das Unerwartbare, wie es Pennycook (2012) in Anlehnung an Heller (2007) formuliert und weiter denkt „unexpect the expected“.

\section{Literatur}

Blommaert, Jan (2013): Ethnography, Superdiversity and Linguistic Landscapes. Chronicles of Complexity. Bristol - Buffalo - Toronto: Multilingual Matters.

Blommaert, Jan (2016) The conservative turn in linguistic landscape studies. In: Ctrl+Alt+Dem. Research on alternative democratic life in Europe. https://alternative-democracy-research. org/2016/01/05/the-conservative-turn-in-linguistic-landscape-studies/ (20. 11. 2019).

Blommaert, Jan (2018): Are chronotopes helpful? In: Working Papers in Urban Language and Literacies 243. Vgl. online: Are chronotopes helpful? In: Ctrl+Alt+Dem. Research on alternative democratic life in Europe. https://alternative-democracy-research.org/2018/06/22/are-chronotopes-helpful/ (20. 11. 2019).

Blommaert, Jan - Backus, Ad (2013): Superdiverse repertoires and the Individual. In: Saint-Georges, Ingrid de - Weber, Jean-Jacques: Multilingualism and Multimodality. Current Challenges for Educational Studies. Rotterdam - Boston - Taiepei: Sense Publishers, S. 11-32.

Busch, Brigitta (2013): Mehrsprachigkeit. Wien: facultas wuv.

Hall, T. Alan (2000): Phonologie: eine Einführung. Berlin, De Gruyter.

Hayes, Bruce (2009): Introductory phonology. Oxford, Wiley-Blackwell.

Heller Monica (2007): The future of ,bilingualism‘. In: Heller, Monica (hrsg.): Bilingualism. A Social Approach. New York: Palgrave Macmillan, S. 340-345.

Pennycook, Alastair (2012): Language and Mobility. Unexpected Places. Bristol - Buffalo - Toronto: Multilingual Matters.

Pennycook, Alastair - Otsuji, Emi (2015): Metrolingualism. Language in the City. Oxon - New York: Routledge.

Stadt Wien (hrsg.) (2019): Migrantinnen und Migranten in Wien 2019. Stadt Wien - Integration und Diversität. Wien: Stadt Wien.

Mag. Thomas Fritz, PhD / thomas.fritz@univie.ac.at

Universität Wien, Philologisch-Kulturwissenschaftliche Fakultät, Institut für Germanistik Universitätsring 1, 1010 Wien, AT 
